\title{
Clinical and laboratory findings in referrals for mitochondrial DNA analysis
}

\author{
P J Lamont, R Surtees, C E Woodward, J V Leonard, N W Wood, A E Harding
}

\begin{abstract}
Background-Increasingly, mutations of mitochondrial DNA (mtDNA) are being considered when investigating the aetiology of neurological diseases in childhood. However, they are often difficult to predict clinically.

Method-Mitochondrial DNA analysis was carried out on 190 children from 1992 to 1996. Most patients were screened for large scale rearrangements and point mutations at nucleotide positions 3243, 3271, 8344, and 8993.

Results-Mutations were found in only 15 patients $(7.9 \%)$ and were either large scale rearrangements (seven patients) or point mutations at nucleotide position 3243 (eight patients). Other point mutations were screened for depending on the clinical picture. The age of symptom onset was significantly older in children with an mtDNA mutation (mean 7.0 years) compared with children without a mutation (mean 2.8 years). Neither Leigh's syndrome (28 cases) nor severe infantile lactic acidosis (12 cases) was associated with mtDNA mutation. Only three clinical features were significantly associated with an mtDNA mutation: progressive external ophthalmoplegia, myopathy, and pigmentary retinopathy. Family history was valuable: the point mutation at nucleotide 3243 (but not the large scale rearrangements) was associated with maternal inheritance; and consanguinity was not associated with mtDNA mutations. The only investigation that provided specific evidence of an underlying mtDNA mutation was histochemical staining of muscle biopsy specimens. The large scale mutations associated with Kearns-Sayre syndrome and progressive external ophthalmoplegia were found in DNA from muscle only, not leucocyte DNA; whereas point mutations were found in leucocyte DNA.
\end{abstract}

Conclusions-Even among children seen at a neurogenetic referral centre, $m$ tDNA mutations were very uncommon. Muscle biopsy was the only investigation to provide evidence of mtDNA abnormality. (Arch Dis Child 1998;79:22-27)

Keywords: mitochondrial DNA mutation; mitochondrial cytopathy; Leigh's syndrome; severe infantile lactic acidosis

Dr Harding has died since the submission of this article.

Correspondence to: Dr Wood.

Accepted 9 February 1998
Pathogenic mitochondrial DNA (mtDNA) mutation has been implicated in the aetiology of a variety of childhood neurological diseases, including Leigh's syndrome ${ }^{1}$, myoclonic epilepsy, ${ }^{23}$ myopathy, ${ }^{4}$ and fatal infantile encephalopathy. ${ }^{5}$ Although specific phenotypes have been described for each of the mtDNA mutations, significant overlap in clinical manifestations occurs. ${ }^{6}$ Thus, the presence of an mtDNA mutation can be difficult to predict, and this can be further complicated by oligosymptomatic or non-specific initial presentation.

This is a review of the first 190 children whose mtDNA was analysed by our molecular biology service laboratory as part of the investigation of their symptoms. It evaluates whether any factors were predictive of a mutation and it aims to provide the physician with practical guidance about when mtDNA analysis should be requested.

\section{Methods}

Patients whose mtDNA had been analysed between 1992 and 1996 and who were aged 18 years or less were selected from the records of the Neurogenetics Laboratory, Institute of Neurology, Queen Square. A group of 190, representing all those referred from either the Hospital for Sick Children, Great Ormond Street, or the Hospital for Neurology and Neurosurgery in Queen Square were chosen because a full clinical and investigative history was available in these children. Charts were reviewed to determine the clinical details.

The mtDNA mutation screen was performed as part of the routine neurogenetics service and was determined by clinical presentation. Manifestations suggestive of the mitochondrial encephalopathy, lactic acidosis and stroke-like episodes (MELAS) syndrome (stroke-like episodes, deafness, ataxia, and limb weakness) led to screening for point mutations at nucleotide (nt) 3243 and nt 3271, whereas manifestations suggestive of myoclonic epilepsy with ragged red fibres (MERRF) (myoclonus, ataxia, seizures, and limb weakness) were screened for point mutations at nt 8344 and nt 8356 . However, because of the large overlap between syndromes, many patients were screened for all of these point mutations. Children referred with Leigh's syndrome were screened for point mutations at nt 8993 and nt 8344 , as well as large scale rearrangements. Manifestations resembling either Pearson's syndrome (sideroblastic anaemia and exocrine and/or endocrine pancreatic insufficiency) or Kearns-Sayre syndrome (KSS) (progressive external ophthalmoplegia, pigmentary retinopathy, limb weakness, heart block, and ataxia) were screened for a large scale rearrangement, and in the latter syndrome, a point mutation at 
Table 1 Screening for mtDNA mutations and rearrangement

\begin{tabular}{lc}
\hline Mutation & $\begin{array}{l}\text { Number of times } \\
\text { screened }\end{array}$ \\
\hline 3243 & 122 \\
8344 & 103 \\
8993 & 90 \\
del/dup & 50 \\
3271 & 34 \\
8356 & 20 \\
3251 & 7 \\
3260 & 6 \\
\hline
\end{tabular}

Other mutations screened for at lower frequency include: $n$ 4317, 14459, 14708, 15990 3252 , and 4269 .
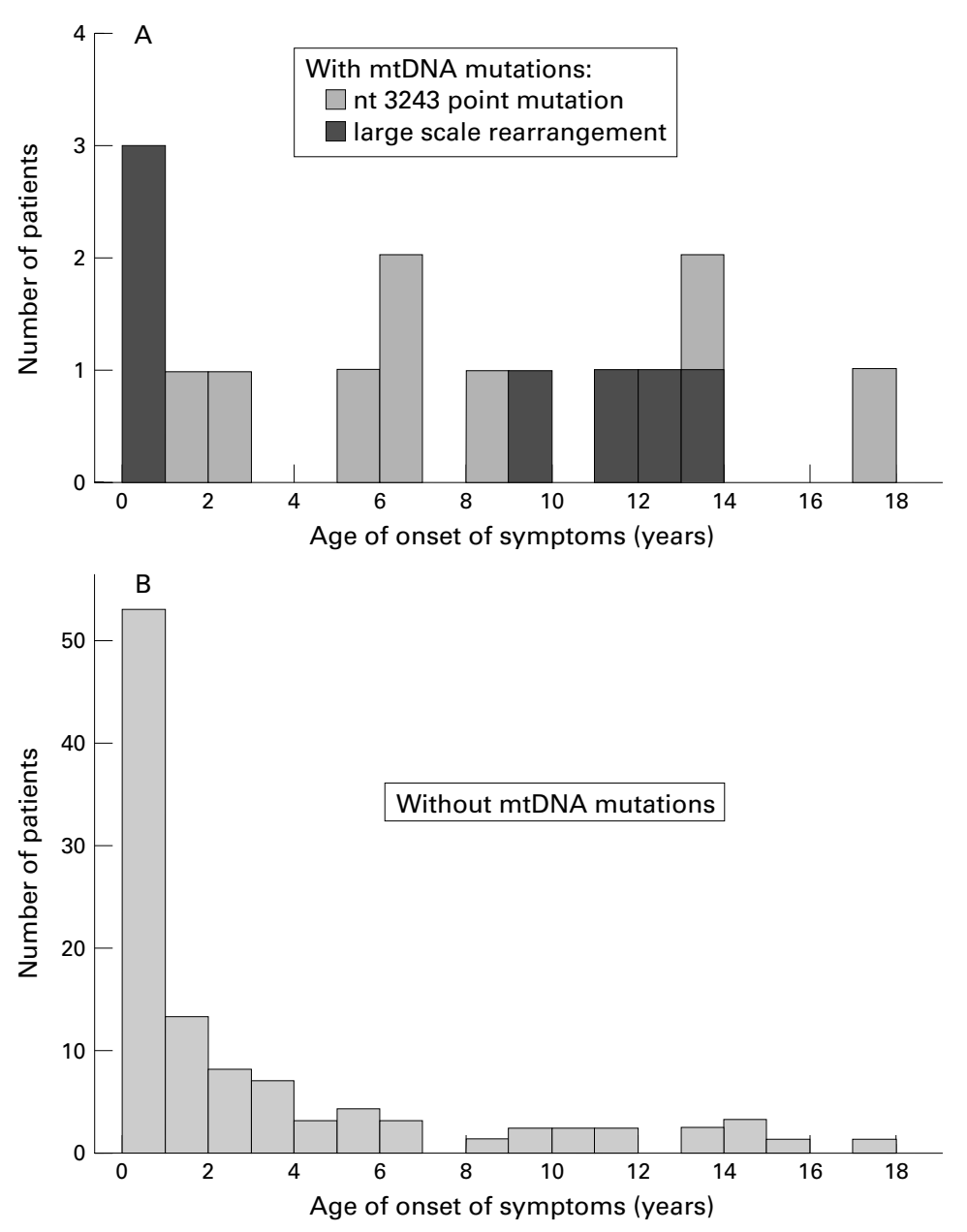

Figure 1 Age at onset of symptoms for children with and without mtDNA mutations.

nt 3243. Other mutations were looked for when a more specific clinical picture presented, such as a point mutation at nt 14459 with early onset generalised dystonia. The mutation screen is summarised in table 1. Depletion of mtDNA was not investigated.

Using standard techniques, DNA was extracted from blood in all patients and from muscle in 77 patients (44\%). For detection of large scale rearrangements, the mtDNA samples were digested with a variety of restriction endonucleases $^{78}$ under conditions recommended by the manufacturer, with the addition of bovine serum albumin (BSA) and spermidine. The digested DNA fragments were separated by horizontal agarose gel (0.8-1.6\%) electrophoresis and transferred to a nylon membrane by Southern blotting. Prehybridisation and hybridisation were performed as described previously, ${ }^{78}$ and the mtDNA fragments were visualised by autoradiography for 24-72 hours at $-70^{\circ} \mathrm{C}$. For detection of point mutations, mtDNA in the appropriate region was amplified by means of the polymerase chain reaction (PCR) as described previously. ${ }^{7}$ The PCR product was digested using 10 units each of the appropriate restriction endonuclease. The digest products were separated on an ethidium bromide stained agarose gel $(3.2 \%)$ and visualised by ultraviolet transillumination.
Contingency tables were constructed and Fisher's exact test and the Mann-Whitney U test were used to test for significance.

\section{Results}

OTHER DIAGNOSES

Alternative diagnoses were made subsequent to referral in 13 cases, and these included Krabbe disease, glucose-6-phosphate dehydrogenase deficiency, Rett syndrome, autosomal dominant hypoparathyroidism, McArdle disease, Rasmussen encephalitis, dermatomyositis, sialidosis, Brown-Vialetto-Van Laere syndrome, Unverricht Lundborg syndrome, and Sandhoff disease. These cases were excluded from further analysis, leaving a group of 177 patients.

\section{mIDNA MUTATIONS FOUND: CLINICAL}

PHENOTYPES

mtDNA mutations were found in 15 of the remaining 177 patients. Eight patients had a point mutation at nt 3243 and seven patients had large scale rearrangements.

Of the eight children with the nt 3243 point mutation, five had stroke-like episodes. Three of the five children were developmentally and neurologically normal before the first strokelike episode. The remaining two children had antecedent problems: one had mild myopathy, one had developmental delay, and both were of short stature. Three of the eight children with the nt 3243 point mutation had no stroke-like episodes. Two of these children had a clinical presentation suggestive of KSS, with ptosis, mild proximal myopathy, progressive external ophthalmoplegia, and ragged red fibres on muscle biopsy. One of the three children had early developmental delay, poor growth, seizures and ataxia, which progressed to dementia, optic atrophy, and pyramidal tract signs by 14 years of age, a presentation suggestive of the syndrome of myoclonic epilepsy with ragged red fibres (MERRF). ${ }^{9}$

Three of the seven children with large scale rearrangements of mtDNA detected in blood had Pearson's marrow/pancreas syndrome. ${ }^{10}$ One child was detected at 6 months of age, while still requiring transfusions to maintain haemoglobin levels, and subsequently developed exocrine and endocrine pancreatic failure. One child was referred at 10 years of age when the haematological problems had settled but other symptoms had appeared, including renal impairment and progressive neurological deterioration with myopathy and ptosis. The third child had a history of anaemia from birth, requiring multiple blood transfusions. At 2 years of age myopathy, ptosis, and lactic acidosis developed, with multiple endocrine deficiencies, renal tubulopathy, and retinopathy developing over the next two years. One of these children had the so-called "common deletion", which is 4977 base pairs long and removes DNA between the ATPase 8 gene and the ND 5 gene (subunit 5 of $\mathrm{NADH}$ ubiquinone reductase). The other two children had slightly larger size deletions of 5390 base pairs (break points 7549-12 939) and 6003 base pairs (break points 7981-13 984). 
Table 2 Clinical and laboratory features according to mtDNA analysis

\begin{tabular}{lcll}
\hline & $\begin{array}{c}\text { +ve mtDNA mutation } \\
\%(n)\end{array}$ & $\begin{array}{l}-v e \text { mtDNA mutation } \\
\%(n)\end{array}$ & p value \\
\hline $\begin{array}{l}\text { Sign/symptom } \\
\text { Myopathy }\end{array}$ & $73 \%(11)$ & $27 \%(39)$ & 0.0002 \\
Ataxia & $47 \%(7)$ & $28 \%(41)$ & NS \\
PEO & $40 \%(6)$ & $<<1 \%(1)$ & $<0.0001$ \\
Stroke-like episodes & $40 \%(6)$ & $20 \%(30)$ & NS \\
$\begin{array}{l}\text { Pigmentary retinopathy } \\
\text { Developmental delay }\end{array}$ & $33 \%(5)$ & $13 \%(19)$ & 0.0354 \\
Seizures & $33 \%(5)$ & $59 \%(87)$ & NS \\
Dystonia & $27 \%(4)$ & $34 \%(50)$ & NS \\
Sensorineural deafness & $13 \%(2)$ & $18 \%(26)$ & NS \\
Investigation (Number positive of number tested) & $7 \%(10)$ & \\
Muscle biopsy & $90 \%(9$ of 10$)$ & $17 \%(12$ of 69$)$ & $<0.0001$ \\
Blood or CSF lactate & $73 \%(11$ of 13$)$ & $45 \%(66$ of 135$)$ & NS \\
MRI of head & $63 \%(5$ of 8$)$ & $37 \%(38$ of 104$)$ & NS \\
\hline
\end{tabular}

$\mathrm{p}$ value are results analysed by Fisher's exact test.

PEO, progressive external ophthalmoplegia; CSF, cerebrospinal fluid; MRI, magnetic resonance imaging.

Three of the seven children with large scale rearrangements had KSS as defined by Berenberg and colleagues, ${ }^{11}$ with ptosis, progressive external ophthalmoplegia, a pigmentary retinopathy, and proximal myopathy. Ragged red fibres were present in two patients and cytochrome oxidase negative fibres were seen in all three on muscle biopsy. The large scale rearrangement was detected in mtDNA from muscle in all three patients but was not detected in blood. None of these children had the common deletion. The size of the deletions were 6278 base pairs (break points 7398 13676 ), 9665 base pairs (break points 590715 572), and 8788 base pairs (break points 6543-15 331). A seventh child had the common deletion with the onset of ptosis at the age of 11 years, which progressed to significant external ophthalmoplegia with mild myopathy affecting the shoulder girdle muscles over the next five years. All seven children had a variable percentage of duplicated species detected, but in all the children this was less than $5 \%$ of the total rearranged species.

AGE OF ONSET OF SYMPTOMS

The median age of onset in those children found to have an mtDNA mutation was 6

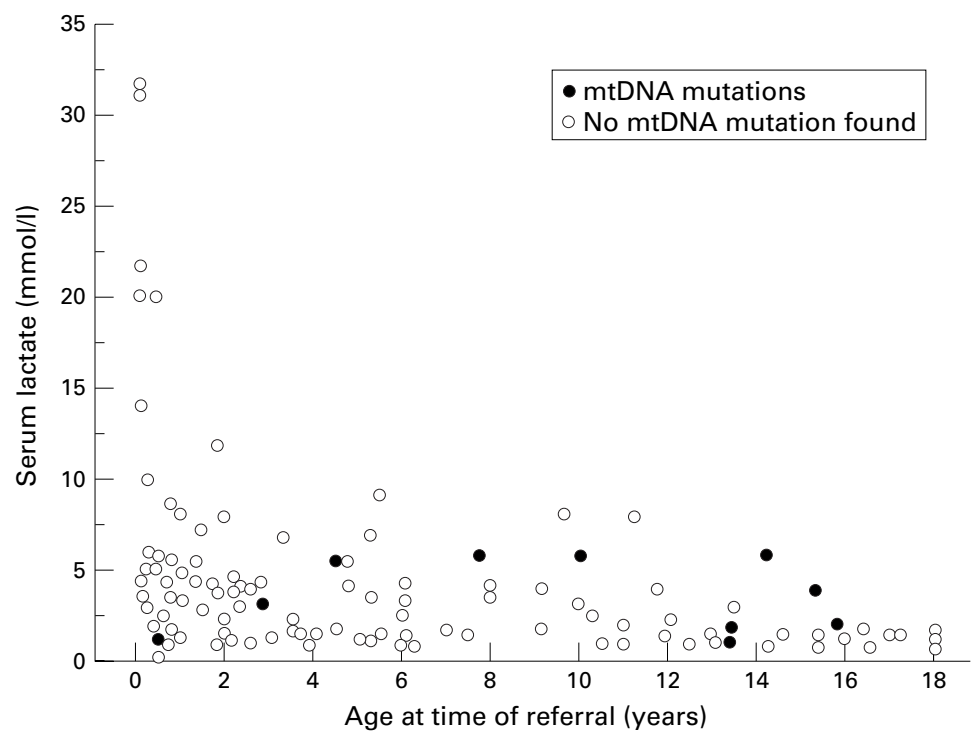

Figure 2 Serum lactate concentrations compared with age at time of referral. years, compared with 1 year in the group without an identified mutation, a significant difference $(p<0.006)$. In part, this is because of the large number of children under the age of 1 year at onset of symptoms in whom no mutation was identified. In contrast, the age of onset of symptoms of those children who did have a mutation was more evenly spread from the first year of life to 18 years, as illustrated in fig 1 .

SURVEY OF CLINICAL FEATURES

Table 2 lists the clinical signs, with the group of 177 patients split according to the results of the mtDNA analysis. The most common features in the group with mtDNA mutation were myopathy, ataxia, progressive external ophthalmoplegia, and stroke-like episodes. In children without mtDNA mutation, developmental delay was the most common symptom, followed by seizures, ataxia, and myopathy. However, the only features seen significantly more often in the group with mtDNA mutations were myopathy $(\mathrm{p}=0.0002)$, progressive external ophthalmoplegia ( $\mathrm{p}<0.0001)$, and pigmentary retinopathy $(\mathrm{p}=0.0354)$.

\section{INVESTIGATIONS}

Serum lactate was measured in all but 14 children and lactic acidosis was found more commonly in those children with a detectable mtDNA mutation than in those without $(78 \%$ compared with 44\%). However, there was a large overlap between blood concentrations of lactate in the two groups (fig 2).

Muscle biopsy was not performed in all cases: it was performed in $64 \%$ with and $49 \%$ without identified mtDNA mutations. Of those children undergoing biopsy, ragged red or cytochrome oxidase negative fibres were found in $89 \%$ of mtDNA mutation positive cases, but only in $17 \%$ of negative cases ( $p<0.0001)$.

Magnetic resonance imaging (MRI) of the brain was performed in 112 of the 177 cases $(71 \%)$. Abnormalities were found in $63 \%$ and $37 \%$ of children with and without an identified mtDNA mutation, respectively (not significant). MRI was performed in five of eight children with the point mutation at nt 3243: one child was within "normal"; three children had appearances resembling infarction in a distribution not conforming to the vascular territory of a large vessel, principally occipito-parietal areas; and one child had gross cerebral atrophy. MRI was performed at the time of an acute stroke-like episode in all but the last child, in whom it was performed because of a subacute deterioration in his general condition. MRI was performed in two of the seven children who had large scale rearrangements: one had mild cerebral atrophy and the other had a diffuse high signal on T2 weighted imaging from subcortical white matter, external capsules, putamina, tectum, tegmentum, and cerebellar peduncles. The abnormalities seen in the children without identified mtDNA mutations were either infarction in a typical vascular territory or the appearances characteristic of Leigh's syndrome. 
LEIGH'S SYNDROME AND SEVERE INFANTILE LACTIC ACIDOSIS

Leigh's syndrome was diagnosed in 28 cases on the basis of the following criteria: an acute or subacute presentation on a background of normal or near normal antecedent development, symptoms or signs referrable to the brainstem, a raised cerebral spinal fluid lactate, and the presence of high signal lesions on T2 weighted MR imaging in the putamen, caudate, thalamus, and dorsal brainstem. ${ }^{12-14}$ Biochemical analysis of cultured fibroblasts revealed a deficiency of complex I and III in one child. Cytochrome oxidase negative muscle fibres were seen in one patient and reduced cytochrome oxidase activity was seen in three patients. However, none of the children with a clinical diagnosis of Leigh's syndrome had the mtDNA mutations reported previously at nt $8993,{ }^{115}$ or nt $8344,{ }^{3}$ or a large scale single deletion. ${ }^{16}$ Other common mutations, such as a point mutation at nt 3243, were not detected.

Severe infantile lactic acidosis was diagnosed in 12 infants, based on early onset of hypoglycaemia and acidosis, with a normal ammonia concentration and raised lactate. ${ }^{17}$ These children were aged 1 week to 12 months at the time of testing, but none had a detectable mtDNA mutation.

\section{FAMILY HISTORY}

Maternal inheritance was established in seven of eight children with the point mutation at $\mathrm{nt}$ 3243. In one case, the diagnosis of the mutation was made first in the mother, who had the onset of ptosis in her early twenties, followed by proximal muscle weakness, restricted eye movements, and exercise intolerance. The mother's mother and brother had died prematurely with a cardiomyopathy. The identification of the point mutation in the child followed diagnosis in the mother. In the remaining six children, maternal inheritance was recognised subsequent to the nt 3243 mutation being found in the child. Two mothers had stroke-like episodes at a young age, leading to death in one but with the other making a good recovery. Another mother had sensorineural deafness and short stature, another had diabetes, a third had exercise intolerance and muscle cramps, and the final one had mild ptosis. In all six mothers, the nt 3243 point mutation was identified in blood. Maternal inheritance was not established in the last of the eight children with the 3243 point mutation because the family refused further investigation. Maternal inheritance was not found in children with large scale rearrangements of mtDNA, in that none had a history of clinically affected relatives, and the mother of each child with an identified mtDNA rearrangement was studied but no mtDNA mutation was found. Consanguinity (parents were first cousins) was present in the family history of 28 children $(17 \%)$. This was not associated with detectable mtDNA mutations. Of the 162 remaining cases, there was only one case with a maternal history $(\mathrm{p}<0.0001)$. This child had multiple symmetrical lipomatosis and hepatic involvement and his mother had lipomatosis.
Ten different mutations were screened for but none were found. Investigation of this child's mtDNA is still being carried out.

\section{Discussion}

The purpose of our study was to identify which characteristics were predictive of an mtDNA mutation in this group of 190 children. Despite being a selected group, mutations/rearrangements were found in only $7.9 \%$, suggesting that mtDNA mutations are an uncommon cause of neurological symptoms in children. Although many mtDNA mutations are described, ${ }^{18}$ only two mutations were found in our study, indicating that many reported mutations are "novel" and confined to one or very few families.

The diagnoses of Leigh's syndrome and severe infantile lactic acidosis were not associated with mtDNA abnormalities in our study. Several mtDNA mutations have been described in association with Leigh's syndrome, including point mutations at nt 8993 (both T to $\mathrm{G}$ and $\mathrm{T}$ to $\mathrm{C}),{ }^{15}{ }^{19}{ }^{20}$ a deletion, ${ }^{16}{ }^{21}$ and a point mutation at nt $8344 .^{3}$ Although a $\mathrm{T}$ to $\mathrm{G}$ mutation at nt 8993 was suggested as a "common" cause of Leigh's syndrome, ${ }^{22}$ our study did not find a mutation at that nucleotide position or any other. Thus, mtDNA mutation is not a frequent cause of Leigh's syndrome in all populations. Two infants with a fatal multisystem disorder of infancy in association with hyperlactataemia have been described in association with point mutations of mtDNA in tRNA $^{\text {thr }} .^{5}$ Despite the assertion of these authors that such mutations affecting the accuracy of reading function of mtDNA tRNA genes might be a frequent cause of fatal infantile lactic acidosis, no mtDNA mutations were found in the 12 infants with severe infantile lactic acidosis in our study.

In the eight children with a point mutation at nt 3243, the mutation was detectable in the mtDNA of their leucocytes. It has been noted previously that analysis of blood mtDNA for the nt 8344 and nt 3243 mutations can be used as an effective screening test for mitochondrial disease in patients with myoclonic epilepsy, ataxia, or early/atypical stroke. ${ }^{23}$ The rearranged species found in the three children with Pearson's syndrome were also detectable in leucocyte mtDNA. However, in the three cases with KSS and the one case with "progressive external ophthalmoplegia plus" syndrome, the rearrangement was detectable in their muscle mtDNA but not in leucocyte mtDNA. This is important for the clinician, because when the phenotype is of KSS or progressive external ophthalmoplegia, mtDNA mutation cannot be excluded as a cause for disease unless DNA from muscle has been tested. Previous studies of the extramuscular distribution of heteroplasmic mtDNA deletions in patients with both progressive external ophthalmoplegia and KSS have shown that post-mitotic tissues, such as muscle, liver, kidney, and brain, are positive for the deletion but lymphocytes or fibroblasts are not. ${ }^{24}$ In our study, there was no apparent 
correlation between the size or site of mtDNA deletion and the type or severity of presentation.

Mitochondrial DNA mutations presenting in the infant can manifest as Pearson's syndrome, Leigh's syndrome, encephalomyopathies, or more generalised multisystem diseases. Initial presentations alter later in childhood, and include a predominant ocular myopathy with or without mild limb involvement, a predominant limb myopathy, or the "specific" encephalomyopathies (KSS, MERRF, or MELAS). The three children in our study with onset in infancy had Pearson's syndrome. The children over the age of 1 year at onset had KSS, an ocular myopathy, MELAS, or MERRF. However, the clinical picture was not always predictive of the mutation found, and a large degree of overlap in clinical picture occurred, a feature recognised previously. ${ }^{25-27} \mathrm{~A}$ third of the 190 patients referred were under the age of 1 year at the time of their initial symptoms, but the median age of onset in the group with identified mtDNA mutations was 6 years. Thus, mtDNA mutations present less frequently in infancy or early childhood.

Clinical features in this group of children that were significantly associated with an underlying mtDNA abnormality were progressive external ophthalmoplegia, myopathy, and pigmentary retinopathy. This finding reflects both the frequency with which they are found in mitochondrial encephalomyopathies and also their specificity for those diseases. In a study of 66 patients with histologically defined mitochondrial myopathy, of whom $61 \%$ were under 20 years of age at onset, 52 patients had ptosis, external ophthalmoplegia, or both; 58 had limb weakness; and 24 had pigmentary retinopathy. ${ }^{28}$ The other relatively specific clinical presentation is that of Pearson's marrow/pancreas syndrome, although recently a case without marrow involvement has been described. ${ }^{29}$

A positive matrilineal family history was present in seven of eight children with the 3243 tRNA $^{\mathrm{Leu}(\mathrm{UUR})} \mathrm{A}$ to $\mathrm{G}$ mutation. It is common to have a matrilineal family history with point mutations of mtDNA, although an asymptomatic mother can carry the mutation. ${ }^{3}$ In the six children with a large scale rearrangement the family history was negative. This agrees with previous work ${ }^{27}$ and might indicate that the deletion is a consequence of mutation within the oocyte or zygote. Consanguinity slightly increases the risk of an autosomal recessive disorder. Mutations of mtDNA are most commonly associated with either a matrilineal family history or with sporadic occurrence, with the rare exception of nuclear encoded multiple deletions. ${ }^{30}$ Thus, it is not surprising that in our study consanguinity was not associated with mtDNA mutations.

Muscle biopsy was the only investigation that provided evidence of an underlying mtDNA abnormality, in the form of ragged red fibres or cytochrome oxidase negative fibres on histochemical staining. Other investigations thought to be strong indicators of underlying mitochon- drial disease, such as "typical" changes on MRI, were not seen significantly more often in those children with positive mtDNA mutation. Although lactic acidosis was seen more often in the positive mutation group, the overlap between the two groups meant it was not useful as a predictive test (fig 2). Thus, if there is a strong clinical suspicion of mtDNA encephalomyopathy, a muscle biopsy might give more specific histochemical, biochemical, and molecular biological evidence. However, it should be emphasised that some mtDNA point mutations within protein coding genes (such as the "NARP" mutation (neurogenic ataxia and retinitis pigmentosa) at nt $8993^{7}$ ) are not associated with ragged red fibres.

Therefore, some clinical and laboratory features were indicative of an underlying mtDNA mutation, such as the presence of a myopathy, progressive external ophthalmoplegia, pigmentary retinopathy, or maternal inheritance on family history. A muscle biopsy should be considered because it might give specific histochemical evidence. Neither Leigh's syndrome nor severe infantile lactic acidosis were associated with detectable mtDNA abnormalities in our study.

PL was supported by the Commonwealth Scholarship Commission in the United Kingdom. RS was supported by the
Wellcome Trust. The authors thank all the referring clinicians. We very much regret the death of Professor Harding during this study.

1 Tatuch Y, Christodoulou J, Feigenbaum A, et al. Heteroplasmic mtDNA mutation (T-G) at 8993 can cause Leigh disease when the percentage of abnormal mtDNA is high. $\mathrm{Am}$ f Hum Genet 1992;50:852-8.

2 Shoffner J, Lott M, Lezza A, Seibel P, Ballinger S, Wallace D. Myoclonic epilepsy and ragged red fiber disease MERRF) is associated with a mitochondrial DNA tRNALys mutation. Cell 1990;61:931-7.

3 Sweeney M, Hammans S, Duchen L, et al. Mitochondrial DNA mutation underlying Leigh's syndrome: clinical, pathological, biochemical, and genetic studies of a patient presenting with progressive myoclonic epilepsy. 7 Neurol Sci 1994;121:57-65.

4 Ionasescu V, Hart M, DiMauro S, Moraes C. Clinical and morphological features of a myopathy associated with a point mutation in the mitochondrial tRNAPro gene. point mutation in the
Neurology 1994;44:975-7.

5 Yoon K, Aprille J, Ernst S. Mitochondrial tRNAthr mutation in fatal infantile respiratory enzyme deficiency. mutation in fatal infantile respiratory enzyme defim
Biochem Biophys Res Commun 1991;176:1112-15.

6 Jackson M, Schaefer J, Johnson M, Morris A, Turnbull, Bindoff L. Presentation and clinical investigation of mitochondrial respiratory chain disease. A study of 51 patients. Brain 1995;118:339-57.

7 Holt I, Harding A, Petty R, Morgan-Hughes J. A new mitochondrial disease associated with mitochondrial DNA heteroplasmy. Am f Hum Genet 1990;46:428-33.

8 Holt I, Harding A, Morgan-Hughes J. Deletions of muscle mitochondrial DNA in patients with mitochondrial myopathies. Nature 1988;331:717-19.

9 Berkovic S, Carpenter S, Evans A, et al. Myoclonus epilepsy and ragged-red fibres (MERRF). 1. A clinical, pathological, biochemical, magnetic resonance spectrographic and positron emission tomographic study. Brain 1989;112: positron $1231-60$.

10 Rotig A, Colonna M, Bonnefont J, et al. Mitochondrial DNA deletion in Pearson's marrow/pancreas syndrome. Lancet 1989;i:902-3.

11 Berenberg R, Pellock J, DiMauro S. Lumping or splitting? "Ophthalmoplegia-plus" or Kearns-Sayre syndrome? Ann Neurol 1977;1:37-54.

12 Davis P, Hoffman J Jr, Braun I, Ahmann P, Krawiecki N. MR of Leigh's disease (subacute necrotizing encephalomyelopathy). Am f Neuroradiol 1987;8:71-5.

13 Geyer C, Sartor K, Prensky A, Abramson C, Hodges F, Gado $M$. Leigh disease (subacute necrotizing encephalomyelopathy): CT and MR in five cases. 7 Comp Assist Tomog 1988;12:40-4.

14 Pincus J. Subacute necrotizing encephalomyelopathy (Leigh's disease): a consideration of clinical features and etiology. Dev Med Child Neurol 1972;14:87-101.

15 de Vries D, van Engelen B, Gabreels F, Ruitenbeek W, van Oost B. A second missense mutation in the mitochondrial ATPase 6 gene in Leigh's syndrome. Ann Neurol 1993;34: ATPase 6 
16 Yamamoto M, Clemens P, Engel A. Mitochondrial DNA deletions in mitochondrial cygentions in 19 deletions in mitochondrial cytopathies:
patients. Neurology 1991;41:1822-8.

17 Aicardi J. Diseases of the nervous system in childhood. London: MacKeith Press, 1992:1408.

18 Anon. Mitochondrial encephalomyopathies: gene mutation. Neuromuscular Disord 1995;5:v-vi.

19 Shoffner J, Fernhoff P, Krawiecki N. Subacute necrotizing encephalopathy: oxidative phosphorylation defects and the ATPase 6 point mutation. Neurology 1992;42:2168-74.

20 Ciafaloni E, Santorelli F, Shanske S. Maternally inherited Leigh's syndrome. 7 Pediatr 1993;122:419-22.

21 Somer H, Suomalainen A, Haltia M, Peltonen L. Deletion of mitochondrial DNA in the adult form of Leigh's syndrome. Neurology 1991;41(suppl 1):208.

22 Santorelli F, Shanske S, Macaya A, DeVivo D, DiMauro S. The mutation at nt 8993 of mitochondrial DNA is a common cause of Leigh's syndrome. Ann Neurol 1993;34:82734 .

23 Hammans S, Sweeney M, Brockington M, Morgan-Hughes J, Harding A. Mitochondrial encephalopathies: molecular
genetic diagnosis from blood samples. Lancet 1991;337: genetic diagn
24 Zeviani M, Gellera C, Pannacci M, et al. Tissue distribution and transmission of mitochondrial DNA deletions in mitochondrial myopathies. Ann Neurol 1990;28:94-7.

25 Byrne E, Trounce I, Dennett X. Progression from MERRF to MELAS phenotype in a patient with combined respiratory complex I and IV deficiencies. 7 Neurol Sci 1988;88:327-38.

26 Hammans S, Morgan-Hughes J. Mitochondrial myopathies: clinical features, investigation, treatment and genetic counselling. Mitochondrial Disorders in Neurology 1994;1:49-74.

27 Holt I, Harding A, Cooper J, et al. Mitochondrial myopathies: clinical and biochemical features of 30 patients with major deletions of muscle mitochondrial DNA. Ann Neurol 1989;26:699-708.

28 Petty R, Harding A, Morgan-Hughes J. The clinical features of mitochondrial myopathy. Brain 1986;109:915-38.

29 Morris A, Lamont P, Clayton P. Pearson's syndrome without marrow involvement. Arch Dis Child 1997;77:56-7.

30 Zeviani M, Servidei S, Gellera C, Bertini E, DiMauro S, DiDonato S. An autosomal dominant disorder with multiple deletions of mitochondrial DNA starting at the D-loop ple deletions of mitochondrial DNA
region. Nature 1989;339:309-11. 